Z Rheumatol 2011 · 70:185

DOI 10.1007/s00393-010-0712-3

Online publiziert: 27. Februar 2011

(c) Springer-Verlag 2011

\author{
F. Moosig 1 - O. Distler ${ }^{2}$ \\ ${ }^{1}$ Poliklinik für Rheumatologie, Universitätsklinikum Schleswig-Holstein \\ und Klinikum Bad Bramstedt \\ ${ }^{2}$ Rheumaklinik und Zentrum für experimentelle Rheumatologie, \\ UniversitätsSpital Zürich
}

\title{
Genetik in der Rheumatologie
}

Im Bereich der Molekulargenetik haben die letzten Jahre rasante technische Fortschritte erbracht. Das Humane Genomprojekt, also die vollständige Sequenzierung eines individuellen Genoms, startete 1990, nahm bis zur ersten Verkündigung der vollständigen Sequenz etwa 11 Jahre in Anspruch, erfolgte unter Einbeziehung mehrerer tausend Wissenschaftler und kostete mehr als 10 Mrd. US\$. Die vergleichbare Sequenzierleistung ist heute quasi über Nacht für einige hundert US\$ verfügbar. Eine entsprechende Flut an Daten wurde und wird daher auch bei rheumatischen Erkrankungen generiert. Das verbreitete Vorurteil, dass die enorme Menge und Komplexität der Daten bisher eine praktische Nutzung bzw. einen echten Erkenntnisgewinn nicht zulasse, wird durch die hier vorgelegten Arbeiten zum Schwerpunktthema eindrucksvoll widerlegt.

\section{(7) Analysen des individuellen Genoms werden in Zukunft einen Anteil an der personalisierten Medizin haben}

U. Wagner stellt am Beispiel der rheumatoiden Arthritis dar, dass genomweite Assoziationsstudien (GWAS) nicht nur bekannte genetische Zusammenhänge (z. B. HLA-DR4) bestätigen konnten, sondern zusätzliche genetische Risikokonstellationen identifizierten, die auch Hinweise auf die Immunpathologie im Hinblick auf die beteiligten Zellen und Signalwege geben. Erste Ansätze, auf der Grundlage solcher genetischer Untersuchungen das potenzielle Ansprechen auf Therapien vorherzusagen, werden erkennbar. Somit werden Analysen des individuellen Genoms in Zukunft einen Anteil an der personalisierten Medizin haben.

Im Beitrag von I. Melchers wird dargestellt, dass genetische Untersuchungen auch bei seltenen Erkrankungen, hier den Kollagenosen, möglich sind und bereits erfolgreich durchgeführt wurden. Bei den Kollagenosen konnten in den verschiedenen methodischen Ansätzen Risikogene mit wahrscheinlich funktioneller Relevanz identifiziert werden. Hier wird auch dargestellt, dass es sowohl krankheitsspezifische als auch allgemein zur Autoimmunität prädisponierende genetische Marker gibt. Die weitere Forschung auf diesem Gebiet wird untersuchen müssen, inwieweit die identifizierten genetischen Risikogene nur für die Initiierung der Erkrankung oder auch für die weitere Progression funktionell relevant sind. Hieraus könnten sich dann auch therapeutische Interventionsmöglichkeiten ergeben.

Dass solche Erkenntnisse unter Umständen auch für das Verständnis von Gruppen von Erkrankungen insgesamt bedeutsam sind, zeigt der Beitrag von J. Holle et al., der den Kenntnisstand bei den primär systemischen Vaskulitiden zusammenfasst. Während sich einige allgemein zur Autoimmunität prädisponierende Risikogenvarianten auch hier nachweisen lassen (z. B. PTPN22-Allel), belegen unterschiedliche, zum Teil sogar diametral entgegengesetzte Häufigkeiten bestimmter Risikoallele innerhalb der Gruppe der ANCA-assoziierten Vaskulitiden, dass die verbreitete Zusammenfassung dieser Entitäten für genetische und pathogenetische Untersuchungen zweifelhaft ist.

Das noch verhältnismäßig junge Gebiet der Epigenetik, dargestellt von A. Jüngel und S. Gay, sucht schließlich die Frage zu beantworten, unter welcher übergeordneten Kontrolle die Expression der Gene steht bzw. warum bei identischer Genausstattung eine Körperzelle beispielsweise in eine Muskel- und eine andere in eine Gehirnzelle differenziert. Da die epigenetische Regulation der Genexpression Störungen aufweisen kann, ergeben sich hier auch für rheumatische Erkrankungen ganz neue Ansätze des Krankheitsverständnisses und perspektivisch auch der Therapie.

Ihre

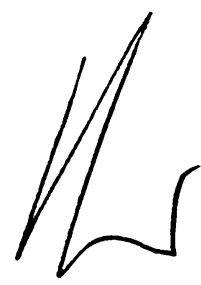

Frank Moosig
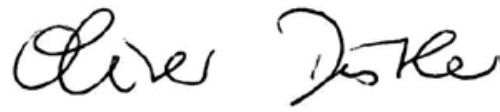

Oliver Distler

\section{Korrespondenzadressen}

PD Dr. F. Moosig

Poliklinik für Rheumatologie, Universitätsklinikum Schleswig-Holstein und Klinikum Bad Bramstedt Oskar-Alexander-Str. 26, 24576 Bad Bramstedt moosig@klinikumbb.de

PD Dr. O. Distler

Rheumaklinik und Zentrum für experimentelle Rheumatologie, UniversitätsSpital Zürich

Gloriastr. 25, 8091 Zürich

Schweiz

oliver.distler@usz.ch 